\title{
SOME NEW RESULTS FOR REICH TYPE MAPPINGS ON CONE $b$-METRIC SPACES OVER BANACH ALGEBRAS
}

\author{
JELENA VUJAKOVIĆ ${ }^{*}$, ABBA AUWALU², VESNA ŠEŠUM-ČAVIĆ3 \\ ${ }^{1}$ Faculty of Sciences and Mathematics, University of Priština, Kosovska Mitrovica, Serbia \\ ${ }^{2}$ Department of Mathematics, Faculty of Sciences and Arts, Near East University, Nicosia, Turkey \\ ${ }^{3}$ Institute of Computer Languages, Vienna University of Technology, Austria
}

\begin{abstract}
The main purpose of this paper is to present some fixed point results concerning the generalized Reich type $\alpha$ admissible mappings in cone $b$-metric spaces over Banach algebras. Our results are significant extensions and generalizations of resent results of N. Hussain at al. (2017) and many well-known results in abundant literature. We also gave an example that confirmed our results.
\end{abstract}

Keywords: Cone $b$-metric space, Banach algebra, $\alpha$-admissible mapping, $\alpha$-regular, Fixed point, $c$-sequence.

\section{INTRODUCTION}

The concept of cone metric space was introduced by Huang and Zhang (2007). They supplanted the set of real numbers in metric space by a complete normed space and proved some fixed point results for different contractive conditions in such a space.

Recently, some scholars (see Du, 2010; Kadelburg et al., 2011) argued that extensions of fixed point results on metric space to cone metric space over complete normed spaces are redundand (not new results). In order to overcome this problem, Liu and $\mathrm{Xu}$ (2013) introduced the notion of a cone metric spaces over Banach algebras and proved that cone metric spaces over Banach algebras are not equivalent to metric spaces in terms of the existence of the fixed points of the generalized Lipschitz mappings. Very recently, Huang and Radenović (2016) introduced the notion of cone $b$-metric space over Banach algebras as a generalization of cone metric space over Banach algebra (see Xu \& Radenović, 2014; Huang \& Radenović, 2015b; Huang \& Xu, 2013; Huang et al., 2017). On the other hand, Samet et al. (2012) introduced the notion of $\alpha$-admissible mappings and proved some fixed point results that generalized several known results of metric spaces. Very recently, Malhottra et al. $(2015 ; 2017)$ used the concept of $\alpha$-admissibility of mappings defined on cone metric space over Banach algebras and proved Banach and Kannan fixed point results for Lipschitz contractions in such spaces. In 2017, Hussaini et al. (2017) used the concept of $\alpha$-admissibility of mappings defined on cone $b$ metric spaces over Banach algebras and proved Banach fixed point results for Lipschitz contractions in such spaces. The Reich contraction was introduced by Reich (1971) as a generalization of the well-known Banach contraction principle and Kannan contraction. In this work, we use the concept of $\alpha$-admissibility of mappings defined on cone $b$-metric space over Banach algebras and proved Reich type fixed point theorems. We give an

* Corresponding author: jelena.vujakovic@pr.ac.rs

MATHEMATICS, COMPUTER SCIENCE AND MECHANICS example to elucidate our results. Our results generalized the recent results of Malhotra et al. (2015; 2017), Hussain et al. (2017), Nieto and Rodríguez-López (2005).

\section{PRELIMINARIES}

It In this section we recall some known definitions and results which will be used.

A real Banach algebra $\mathcal{B}$ is a real Banach space in which an operation of multiplication is defined in the following way: for all $x, y, z \in \mathcal{B}, \alpha \in \mathbb{R}$

1) $\quad(x y) z=x(y z)$

2) $x(y+z)=x y+x z$ and $(x+y) z=x z+y z$,

3) $\quad \alpha(x y)=(\alpha x) y=x(\alpha y)$,

4) $\|x y\| \leq\|x\|\|y\|$.

A subset $\mathcal{K}$ of Banach algebra $\mathcal{B}$ is called a cone if:

1) $\mathcal{K}$ is nonempty, closed and $\{\theta, e\} \subset \mathcal{K}$;

2) $\alpha \mathcal{K}+\beta \mathcal{K} \subset \mathcal{K}$, for all nonnegative $\alpha, \beta \in \mathbb{R}$;

3) $\mathcal{K}^{2}=\mathcal{K} \mathcal{K} \subset \mathcal{K}$;

4) $\mathcal{K} \cap(-\mathcal{K})=\{\theta\}$,

where $\theta$ and $e$ denote the zero and unit elements of Banach algebra $\mathcal{B}$, respectively. For given cone $\mathcal{K} \subset \mathcal{B}$ we define a partial ordering $\preceq$ with respect to $\mathcal{K}$ on following way: $x \preceq y$ if and only if $y-x \in \mathcal{K}$. It is well known that $x \prec y$ stands for $x \preceq y$ and $x \neq y, x \ll y$ stands for $y-x \in$ int $\mathcal{K}$ where int $\mathcal{K}$ means the interior of $\mathcal{K}$. We say that $\mathcal{K}$ is a solid cone if int $\mathcal{K} \neq \varnothing$.

In further work, we always assume that $\mathcal{B}$ is a Banach algebra with a unit $e, \mathcal{K}$ is a solid cone in $\mathcal{B}$ and $\preceq$ is the partial ordering with respect to $\mathcal{K}$.

Definition 2.1 (Huang \& Zhang 2007; Liu \& Xu, 2013) Let $\mathcal{Z}$ be a nonempty set. Suppose that the mapping $\rho: \mathcal{Z} \times \mathcal{Z} \rightarrow \mathcal{B}$ satisfies: 
(C1) $\quad \theta \preceq \rho(x, y)$ for all $x, y \in \mathcal{Z}$ and $\rho(x, y)=\theta$ if and only if $x=y$;

$$
\rho(x, y)=\rho(y, x) \text { for all } x, y \in \mathcal{Z} \text {; }
$$

(C3) $\quad \rho(x, y) \preceq \rho(x, z)+\rho(z, y)$ for all $x, y, z \in \mathcal{Z}$.

Then $\rho$ is called a cone metric on $\mathcal{Z}$ and $(\mathcal{Z}, \rho)$ is called a cone metric space with a Banach algebra $\mathcal{B}$.

Definition 2.2 (Huang \& Radenović, 2015a) Let $\mathcal{Z}$ be a nonempty set, $s \geq 1$ be a constant and $\mathcal{B}$ a Banach algebra. Suppose that the mapping $\rho: \mathcal{Z} \times \mathcal{Z} \rightarrow \mathcal{B}$ satisfies:

(C1) $\quad \theta \preceq \rho(x, y)$ for all $x, y \in \mathcal{Z}$ and $\rho(x, y)=\theta$ if and only if $x=y$;

(C2) $\rho(x, y)=\rho(y, x)$ for all $x, y \in \mathcal{Z}$;

(C3) $\rho(x, y) \preceq s[\rho(x, z)+\rho(z, y)]$ for all $x, y, z \in \mathcal{Z}$.

Then $\rho$ is called a cone $b$-metric on $\mathcal{Z}$ and $(\mathcal{Z}, \rho)$ is called a cone $b$-metric space with a Banach algebra $\mathcal{B}$.

Note that if $s=1$, a cone $b$-metric becomes a cone metric.

Definition 2.3 (Malhotra et al., 2015) Let $(\mathcal{Z}, \rho)$ be a cone $b$ metric space over Banach algebra $\mathcal{B}$ with coefficient $s \geq 1$ and $\left\{z_{i}\right\}$ be a sequence in $(\mathcal{Z}, \rho)$. Then,

1) $\left\{z_{i}\right\}$ converges to $z$ whenever for each $c \in \mathcal{B}$ with $c \gg \theta$ there is a natural number $N$ such that $\rho\left(z_{i}, z\right) \ll c$ for all $i \geq N$. We denote this by $z_{i} \rightarrow z$ $(i \rightarrow \infty)$ or $\lim _{i \rightarrow \infty} z_{i}=z$.

2) $\left\{z_{i}\right\}$ is Cauchy sequence whenever for each $c \in \mathcal{B}$ with $c \gg \theta$ there is a natural number $N$ such that $\rho\left(z_{i}, z_{j}\right) \ll c$ for all $i, j \geq N$.

3) $(\mathcal{Z}, \rho)$ is said to be complete if every Cauchy sequence in $\mathcal{Z}$ is convergent.

Definition 2.4 (Malhotra et al., 2015) A mapping $\mathcal{F}: \mathcal{Z} \rightarrow \mathcal{Z}$ is said to be continuous at $z \in \mathcal{Z}$, if for every sequence $\left\{z_{i}\right\}$ in $(\mathcal{Z}, \rho)$ such that $z_{i} \rightarrow z \quad(i \rightarrow \infty)$, we have $\mathcal{F}_{i} \rightarrow \mathcal{F} z$ $(i \rightarrow \infty)$.

Definition 2.5 (Kadelburg \& Radenović, 2013) Let $\mathcal{K}$ be a solid cone in a Banach algebra $\mathcal{B}$. A sequence $\left\{z_{i}\right\} \subset \mathcal{K}$ is said to be a $c$-sequence if for each $c \gg \theta$ there exists $N \in \mathbb{N}$ such that $z_{i} \ll c$ for all $i>N$.

Lemma 2.6 (Huang \& Radenović, 2015b) If $E$ is a real Banach space with a solid cone $\mathcal{K}$ and $\left\{z_{i}\right\} \subset \mathcal{K}$ be a sequence with $\left\|z_{i}\right\| \rightarrow 0 \quad(i \rightarrow \infty)$, then for each $c \gg \theta$ there exists $N \in \mathbb{N}$ such that for any $i>N$ we have $z_{i} \ll c$.
Lemma 2.7 (Xu \& Radenović, 2015) Let $(\mathcal{Z}, \rho)$ be a complete cone b-metric space over Banach algebra $\mathcal{B}$ with coefficients $s \geq 1$ and $\mathcal{K}$ be the underlying solid cone. Let $\left\{z_{i}\right\}$ be a sequence in $(\mathcal{Z}, \rho)$. If $\left\{z_{i}\right\}$ converges to $z \in \mathcal{Z}$, then:

1) $\quad\left\{\rho\left(z_{i}, z\right)\right\}$ is a $c$-seqauence;

2) for any $j \in \mathbb{N},\left\{\rho\left(z_{i}, z_{i+j}\right)\right\}$ is a c-sequence.

Lemma 2.8 (Kadelburg \& Radenović, 2013) Let $\mathcal{B}$ be a real Banach algebra with a solid cone $\mathcal{K}$ and let $\left\{\alpha_{n}\right\}$ and $\left\{\beta_{n}\right\}$ are sequences in $\mathcal{K}$. If $\left\{\alpha_{n}\right\}$ and $\left\{\beta_{n}\right\}$ are c-sequences and $k_{1}, k_{2} \in \mathcal{K}$ then $\left\{k_{1} \alpha_{n}+k_{2} \beta_{n}\right\}$ is a c-sequence.

Lemma 2.9 (Rudin, 1991; Huang \& Radenović, 2015a) Let $\mathcal{B}$ be a Banach algebra with a unit $e$ and $k \in \mathcal{B}$. Then $\lim _{n \rightarrow \infty}\left\|k^{n}\right\|^{\frac{1}{n}}$ exists and the spectral radijus $\delta(k)$ satisfies

$$
\delta(k)=\lim _{n \rightarrow \infty}\left\|k^{n}\right\|^{\frac{1}{n}}=\inf \left\|k^{n}\right\|^{\frac{1}{n}}
$$

If $\delta(k)<|\lambda|$, then $\lambda e-k$ is invertible in $\mathcal{B}$. Moreover, for complex constant $\lambda$ are $(\lambda e-k)^{-1}=\sum_{j=0}^{\infty} \frac{k^{j}}{\lambda^{j+1}} \quad$ and $\delta\left[(\lambda e-k)^{-1}\right] \leq \frac{1}{|\lambda|-\delta(k)}$

Remark 2.10. (Xu \& Radenović, 2015) If $\delta(k)<1$ then $\left\|k^{i}\right\| \rightarrow 0 \quad(i \rightarrow \infty)$.

Lemma 2.11 (Rudin, 1991) Let $\mathcal{B}$ be a Banach algebra with a unit $e$ and $k_{1}, k_{2} \in \mathcal{B}$. If $k_{1}$ commutes with $k_{2}$, then $\delta\left(k_{1}+k_{2}\right) \leq \delta\left(k_{1}\right)+\delta\left(k_{2}\right)$ and $\delta\left(k_{1} k_{2}\right) \leq \delta\left(k_{1}\right) \delta\left(k_{2}\right)$.

Lemma 2.12 (Kadelburg \& Radenović, 2013) Let $E$ is a real Banach space with a solid cone $\mathcal{K}$.

1) If $a, b, c \in E$ and $a \preceq b \ll c$, then $a \ll c$

2) If $a \in \mathcal{K}$ and $a \ll c$ for each $c \gg \theta$, then $a=\theta$.

3) If $a \preceq k a$, where $a, k \in \mathcal{K}$ and $\delta(k)<1$, then $a=\theta$.

\section{MAIN RESULTS}

First we introduce the notion of $\alpha$-admissible mapping and $\alpha$-regularity in the setting of cone $b$-metric space over Banach algebra $\mathcal{B}$

Definition 3.1 Let $(\mathcal{Z}, \rho)$ be a cone $b$-metric space over Banach algebra $\mathcal{B}$ with coefficients $s \geq 1, \mathcal{K}$ be the underlying solid cone, $\mathcal{F}: \mathcal{Z} \rightarrow \mathcal{Z}$ and $\alpha: \mathcal{Z} \times \mathcal{Z} \rightarrow \mathcal{B}$ be mappings. Then:

1) $\mathcal{F}$ is $\alpha$-admissible mapping if $\alpha(z, y) \succeq s$ implies $\alpha(\mathcal{F} z, \mathcal{F} y) \succeq s$, for all $z, y \in \mathcal{Z}$; 
2) $(\mathcal{Z}, \rho)$ is $\alpha$-regular if for any sequence $\left\{z_{i}\right\}$ in $(\mathcal{Z}, \rho)$, with $\alpha\left(z_{i}, z_{i+1}\right) \succeq s$ for all $i \in \mathbb{N}$ and $z_{i} \rightarrow z_{*} \in \mathcal{Z}$ $(i \rightarrow \infty)$, follows $\alpha\left(z_{i}, z_{*}\right) \succeq s$, for all $i \in \mathbb{N}$.

Now we are able to define generalized Reich type contraction in cone $b$-metric spaces over Banach algebra $\mathcal{B}$.

Definition 3.2 Let $(\mathcal{Z}, \rho)$ be a cone $b$-metric space over Banach algebra $\mathcal{B}$ with coefficient $s \geq 1$, let $\mathcal{K}$ be the underlying solid cone and $\alpha: \mathcal{Z} \times \mathcal{Z} \rightarrow \mathcal{B}$ be a function. The mapping $\mathcal{F}: \mathcal{Z} \rightarrow \mathcal{Z}$ is called generalized Reich type contraction if there exist vectors $\quad v_{k} \in \mathcal{K} \quad(k=1,2,3) \quad$ such $2 s \delta\left(v_{1}\right)+(s+1) \delta\left(v_{2}+v_{3}\right)<2$ and

$$
\rho\left(\mathcal{F}_{z}, \mathcal{F} y\right) \preceq v_{1} \rho(z, y)+v_{2} \rho\left(z, \mathcal{F}_{z}\right)+v_{3} \rho(y, \mathcal{F} y),
$$

for all $z, y \in \mathcal{Z}$ with $\alpha(z, y) \succeq s$.

Now, we shall show that generalized Reich type $\alpha$-admissible contraction mappings on cone $b$-metric spaces over Banach algebra $\mathcal{B}$ has a fixed points.

Theorem 3.3 Let $(\mathcal{Z}, \rho)$ be a complete cone b-metric spaces over Banach algebra $\mathcal{B}$ with coefficient $s \geq 1, \mathcal{K}$ be the underlying solid cone and let $\alpha: \mathcal{Z} \times \mathcal{Z} \rightarrow \mathcal{B}$ be a function. Suppose the mapping $\mathcal{F}: \mathcal{Z} \rightarrow \mathcal{Z}$ is a generalized Reich type contraction with vectors $v_{k} \in \mathcal{K} \quad(k=1,2,3)$ such that $v_{1}$ commutes with $v_{2}+v_{3}$. Suppose that:

1) $\mathcal{F}$ is a $\alpha$-admissible mapping;

2) there exists $z_{0}$ in $\mathcal{Z}$ with $\alpha\left(z, \mathcal{F} z_{0}\right) \succeq s$;

3) $\mathcal{F}$ is continuous or $(\mathcal{Z}, \rho)$ is $\alpha$-regular,

then $\mathcal{F}$ has a fixed point $z_{*}$ in $\mathcal{Z}$.

PROOF. Let $z_{0}$ be an arbitrary point in $\mathcal{Z}$ such that $\alpha\left(z_{0}, \mathcal{F} z_{0}\right) \succeq s$. Define a sequence $\left\{z_{i}\right\}$ in $(\mathcal{Z}, \rho)$ by

$$
z_{i+1}=\mathcal{F}_{z_{i}}=\mathcal{F}^{i+1} z_{0} \text { for all } i \in \mathbb{N} .
$$

If $z_{i+1}=z_{i}$ for some $i \in \mathbb{N}$, then $z_{*}=z_{i}$ is a fixed point of $\mathcal{F}$ and the result is proved. Suppose now that $z_{i+1} \neq z_{i}$ for all $i \in \mathbb{N}$. Since $\mathcal{F}$ is $\alpha$-admissible mapping, we have

$$
\alpha\left(z_{0}, z_{1}\right)=\alpha\left(z_{0}, \mathcal{F} z_{0}\right) \succeq s
$$

Hence,

$$
\alpha\left(\mathcal{F} z_{0}, \mathcal{F}^{2} z_{0}\right)=\alpha\left(z_{1}, z_{2}\right) \succeq s
$$

and by induction we get

$$
\alpha\left(z_{i}, z_{i+1}\right) \succeq s \text {, for all } i \in \mathbb{N} .
$$

Now, according to (1), (2) and (3), we have

$$
\begin{aligned}
& \rho\left(z_{i}, z_{i+1}\right)=\rho\left(\mathcal{F} z_{i-1}, \mathcal{F} z_{i}\right) \\
& \preceq v_{1} \rho\left(z_{i-1}, z_{i}\right)+v_{2} \rho\left(z_{i-1}, \mathcal{F} z_{i-1}\right)+v_{3} \rho\left(z_{i}, \mathcal{F} z_{i}\right) \\
& =\left(v_{1}+v_{2}\right) \rho\left(z_{i-1}, z_{i}\right)+v_{3} \rho\left(z_{i}, z_{i+1}\right) \text {, i.e. } \\
& \qquad\left(e-v_{3}\right) \rho\left(z_{i}, z_{i+1}\right) \preceq\left(v_{1}+v_{2}\right) \rho\left(z_{i-1}, z_{i}\right) .
\end{aligned}
$$

Similarly,

$$
\begin{aligned}
& \rho\left(z_{i+1}, z_{i}\right)=\rho\left(\mathcal{F}_{z_{i}}, \mathcal{F}_{z_{i-1}}\right) \\
& \preceq v_{1} \rho\left(z_{i}, z_{i-1}\right)+v_{2} \rho\left(z_{i}, \mathcal{F}_{z_{i}}\right)+v_{3} \rho\left(z_{i-1}, \mathcal{F} z_{i-1}\right) \\
& =\left(v_{1}+v_{3}\right) \rho\left(z_{i-1}, z_{i}\right)+v_{2} \rho\left(z_{i}, z_{i+1}\right) \text {, i.e. } \\
& \qquad\left(e-v_{2}\right) \rho\left(z_{i}, z_{i+1}\right) \preceq\left(v_{1}+v_{3}\right) \rho\left(z_{i-1}, z_{i}\right) .
\end{aligned}
$$

Adding up (4) and (5), we have

$\left(e-v_{2}-v_{3}\right) \rho\left(z_{i}, z_{i+1}\right) \preceq\left(2 v_{1}+v_{2}+v_{3}\right) \rho\left(z_{i-1}, z_{i}\right)$, i.e.

$$
(e-v) \rho\left(z_{i}, z_{i+1}\right) \preceq\left(2 v_{1}+v\right) \rho\left(z_{i-1}, z_{i}\right),
$$

where $v=v_{2}+v_{3}$.

Note that, $2 \delta(v) \leq(s+1) \delta(v) \leq 2 \delta\left(v_{1}\right)+(s+1) \delta(v)<2$, i.e. $\delta(v)<1<2$. From Lemma 2.9 we conclude that $2 e-v$ is invertible. Moreover, $\quad(2 e-v)^{-1}=\sum_{k=0}^{\infty} \frac{v^{k}}{2^{k+1}} \quad$ and $\delta\left[(2 e-v)^{-1}\right] \leq \frac{1}{2-\delta(v)}$.

Hence, from (6), we have get

$$
\rho\left(z_{i}, z_{i+1}\right) \preceq(2 e-v)^{-1}\left(2 v_{1}+v\right) \rho\left(z_{i-1}, z_{i}\right)=\tau \rho\left(z_{i-1}, z_{i}\right),
$$

where $\tau=(2 e-v)^{-1}\left(2 v_{1}+v\right)$.

Since $v_{1}$ commutes with $v=v_{2}+v_{3}$, it follows that

$$
\begin{aligned}
(2 e-v)^{-1}\left(2 v_{1}+v\right) & =\left(\sum_{k=0}^{\infty} \frac{v^{k}}{2^{k+1}}\right)\left(2 v_{1}+v\right) \\
& =2 v_{1}\left(\sum_{k=0}^{\infty} \frac{v^{k}}{2^{k+1}}\right)+v\left(\sum_{k=0}^{\infty} \frac{v^{k}}{2^{k+1}}\right) \\
& =\left(2 v_{1}+v\right)\left(\sum_{k=0}^{\infty} \frac{v^{k}}{2^{k+1}}\right)=\left(2 v_{1}+v\right)(2 e-v)^{-1} .
\end{aligned}
$$

Thus, $(2 e-v)^{-1}$ commutes with $\left(2 v_{1}+v\right)$. Then by Lemma 2.9 and Lemma 2.11, we have that

$$
\begin{aligned}
\delta(\tau) & =\delta\left[(2 e-v)^{-1}\left(2 v_{1}+v\right)\right] \leq \delta\left[(2 e-v)^{-1}\right] \delta\left(2 v_{1}+v\right) \\
& \leq \frac{1}{2-\delta(v)}\left[2 \delta\left(v_{1}\right)+\delta(v)\right]<\frac{1}{s}
\end{aligned}
$$

Since $\delta(\tau)<\frac{1}{s}$, by Lemma 2.9 and Lemma 2.11, it follows that $e-s \tau$ is invertible. Moreover,

$$
(e-s \tau)^{-1}=\sum_{k=0}^{\infty}(s \tau)^{k} \text { and } \delta\left[(e-s \tau)^{-1}\right] \leq \frac{1}{2-s \delta(\tau)} .
$$


Since $\delta(\tau)<\frac{1}{S}<1$, by Remark 2.10, we have that

$$
\left\|\tau^{i}\right\| \rightarrow 0 \quad(i \rightarrow \infty)
$$

From (7), we get

$$
\begin{aligned}
\rho\left(z_{i}, z_{i+1}\right) & \preceq \tau \rho\left(z_{i-1}, z_{i}\right) \\
& \preceq \tau^{2} \rho\left(z_{i-2}, z_{i-1}\right) \\
& \vdots \\
\rho\left(z_{i}, z_{i+1}\right) & \preceq \tau^{i} \rho\left(z_{0}, z_{1}\right)
\end{aligned}
$$

for all $i \in \mathbb{N}$. Hence, for $i, j \in \mathbb{N}$ with $i<j$, using (8) and (10), we obtain

$$
\begin{aligned}
& \rho\left(z_{i}, z_{j}\right) \preceq s\left[\rho\left(z_{i}, z_{i+1}\right)+\rho\left(z_{i+1}, z_{j}\right)\right] \\
& \preceq s \rho\left(z_{i}, z_{i+1}\right)+s^{2}\left[\rho\left(z_{i+1}, z_{i+2}\right)+\rho\left(z_{i+1}, z_{j}\right)\right] \\
& \vdots \\
& \preceq s \rho\left(z_{i}, z_{i+1}\right)+s^{2} \rho\left(z_{i+1}, z_{i+2}\right)+s^{3} \rho\left(z_{i+1}, z_{i+2}\right) \\
& \quad+\ldots+s^{j-i-1} \rho\left(z_{j-2}, z_{j-1}\right)+s^{j-i-1} \rho\left(z_{j-1}, z_{j}\right) \\
& \preceq s \tau^{i} \rho\left(z_{0}, z_{1}\right)+s^{2} \tau^{i+1} \rho\left(z_{0}, z_{1}\right)+s^{3} \tau^{i+2} \rho\left(z_{0}, z_{1}\right) \\
& \quad+\ldots+s^{j-i-1} \tau^{j-2} \rho\left(z_{0}, z_{1}\right)+s^{j-i-1} \tau^{j-1} \rho\left(z_{0}, z_{1}\right) \\
& \preceq s \tau^{i}\left(e+s \tau+(s \tau)^{2}+\ldots+(s \tau)^{j-i-2}+(s \tau)^{j-i-1}\right) \rho\left(z_{0}, z_{1}\right) \\
& \preceq s \tau^{i}\left(\sum_{k=0}^{\infty}(s \tau)^{k}\right) \rho\left(z_{0}, z_{1}\right)=s \tau^{i}(e-s \tau)^{-1} \rho\left(z_{0}, z_{1}\right) .
\end{aligned}
$$

Obviously, from (9) it follows that

$$
\left\|s \tau^{i}(e-s \tau)^{-1} \rho\left(z_{0}, z_{1}\right)\right\| \rightarrow 0 \quad(i \rightarrow \infty)
$$

and by Lemma 2.6 there exists $N \in \mathbb{N}$ such that for any $c \in \mathcal{B}$, with $c \gg \theta$ is

$$
\rho\left(z_{i}, z_{j}\right) \preceq s \tau^{i}(e-s \tau)^{-1} \rho\left(z_{0}, z_{1}\right) \ll c,
$$

for all $j>i>N$.

This implies that $\left\{z_{i}\right\}$ is Cauchy sequence. Since $(\mathcal{Z}, \rho)$ is complete, there exists $z_{*} \in \mathcal{Z}$ such that $z_{i} \rightarrow z_{*}(i \rightarrow \infty)$.

Supposse that $\mathcal{F}$ is continuous. It follows that $z_{i+1}=\mathcal{F} z_{i} \rightarrow \mathcal{F}_{z_{*}} \quad(i \rightarrow \infty)$. By uniqueness of the limit of sequence in $(\mathcal{Z}, \rho)$, we have that $\mathcal{F}_{z_{*}}=z_{*}$. Thus $z_{*}$ in $\mathcal{Z}$ is a fixed point of $\mathcal{F}$.

On the other hand, if $(\mathcal{Z}, \rho)$ is $\alpha$-regular, by using (3), we have

$$
\alpha\left(z_{i}, z_{*}\right) \succeq s, \text { for all } i \in \mathbb{N} .
$$

Now we show that $z_{*}$ is a fixed point of $\mathcal{F}$, i.e. $\mathcal{F}_{z_{*}}=z_{*}$. From (1) and (11), we obtain

$$
\begin{aligned}
& \rho\left(z_{*}, \mathcal{F}_{z_{*}}\right) \preceq s\left[\rho\left(z_{*}, \mathcal{F}_{z_{i}}\right)+\rho\left(\mathcal{F}_{z_{i}}, \mathcal{F}_{z_{*}}\right)\right] \\
& \preceq s \rho\left(z_{*}, \mathcal{F}_{z_{i}}\right)+s\left[v_{1} \rho\left(z_{i}, z_{*}\right)+v_{2} \rho\left(z_{i}, \mathcal{F} z_{i}\right)+v_{3} \rho\left(z_{*}, \mathcal{F}_{z_{*}}\right)\right] \\
& \preceq s \rho\left(z_{*}, z_{i+1}\right)+s v_{1} \rho\left(z_{i}, z_{*}\right) \\
& \quad+s^{2} v_{2}\left[\rho\left(z_{i}, z_{*}\right)+\rho\left(z_{*}, z_{i+1}\right)\right]+s v_{3} \rho\left(z_{*}, \mathcal{F}_{z_{*}}\right) \\
& =s\left(e+s v_{2}\right) \rho\left(z_{i+1}, z_{*}\right)+s\left(v_{1}+s v_{2}\right) \rho\left(z_{i}, z_{*}\right)+s v_{3} \rho\left(z_{*}, \mathcal{F}_{Z_{*}}\right) \text {, i.e. } \\
& \left(e+s v_{3}\right) \rho\left(z_{*}, \mathcal{F}_{z_{*}}\right) \preceq . \\
& \quad \preceq s\left(e+s v_{2}\right) \rho\left(z_{i+1}, z_{*}\right)+s\left(v_{1}+s v_{2}\right) \rho\left(z_{i}, z_{*}\right) .
\end{aligned}
$$

Similarly,

$$
\begin{aligned}
& \rho\left(\mathcal{F}_{z_{*}}, z_{*}\right) \preceq s\left[\rho\left(\mathcal{F}_{z_{*}}, \mathcal{F}_{z_{i}}\right)+\rho\left(\mathcal{F}_{z_{i}}, z_{*}\right)\right] \\
& \preceq s\left[v_{1} \rho\left(z_{*}, z_{i}\right)+v_{2} \rho\left(z_{*}, \mathcal{F}_{z_{*}}\right)+v_{3} \rho\left(z_{i}, \mathcal{F}_{z_{i}}\right)\right]+\rho\left(\mathcal{F}_{z_{i}}, \mathcal{F}_{z_{*}}\right) \\
& \preceq s v_{1} \rho\left(z_{*}, z_{i}\right)+s v_{2} \rho\left(z_{*}, \mathcal{F}_{z_{*}}\right)+ \\
& \quad+s^{2} v_{3}\left[\rho\left(z_{i}, z_{*}\right)+\rho\left(z_{*}, z_{i+1}\right)\right]+s \rho\left(z_{i+1}, z_{*}\right) \\
& =s v_{2} \rho\left(z_{*}, \mathcal{F}_{z_{*}}\right)+s\left(e+s v_{3}\right) \rho\left(z_{i+1}, z_{*}\right)+s\left(v_{1}+s v_{3}\right) \rho\left(z_{i}, z_{*}\right) \text {, i.e. } \\
& \left(e-s v_{2}\right) \rho\left(z_{*}, \mathcal{F}_{z_{*}}\right) \preceq \\
& \quad \preceq s\left(e+s v_{3}\right) \rho\left(z_{i+1}, z_{*}\right)+s\left(v_{1}+s v_{3}\right) \rho\left(z_{i}, z_{*}\right) .
\end{aligned}
$$

Hence, by combining (12) and (13), we obtain

$$
\begin{gathered}
\begin{aligned}
\left(2 e-s v_{2}-s v_{3}\right) \rho\left(z_{*}, \mathcal{F}_{z_{*}}\right) \preceq & s\left(2 e+s v_{2}+s v_{3}\right) \rho\left(z_{i+1}, z_{*}\right)+ \\
& +s\left(2 v_{1}+s v_{2}+s v_{3}\right) \rho\left(z_{i}, z_{*}\right), \text { i.e. } \\
(2 e-s v) \rho\left(z_{*}, \mathcal{F}_{z_{*}}\right) \preceq & \preceq s(2 e+s v) \rho\left(z_{i+1}, z_{*}\right)+s\left(2 v_{1}+s v\right) \rho\left(z_{i}, z_{*}\right) .
\end{aligned}
\end{gathered}
$$

Note, that

$$
\delta(s v)=s \delta(v) \leq(s+1) \delta(v) \leq 2 s \delta\left(v_{1}\right)+(s+1) \delta(v)<2 .
$$

Since by Lemma $2.9,2 e-s v$ is invertible, it follows from (14) that

$$
\begin{aligned}
& \rho\left(z_{*}, \mathcal{F}_{z_{*}}\right) \preceq \\
& \quad \preceq(2 e-s v)^{-1}\left[\left(2 s e+s^{2} v\right) \rho\left(z_{i+1}, z_{*}\right)+\left(2 s v_{1}+s^{2} v\right) \rho\left(z_{i}, z_{*}\right)\right] .
\end{aligned}
$$

Therefore, by Lemma 2.7 and Lemma 2.8, $\left\{\rho\left(z_{i}, z_{*}\right)\right\}$, $\left\{\rho\left(z_{i+1}, z_{*}\right)\right\}$ and $\left\{\tau_{1} \rho\left(z_{i+1}, z_{*}\right)+\tau_{2} \rho\left(z_{i}, z_{*}\right)\right\}$ are $c$-sequences (we mark $\tau_{1}$ and $\tau_{2}$ respectively by $(2 e-s v)^{-1}\left(2 s e+s^{2} v\right)$, $\left.(2 e-s v)^{-1}\left(2 s v_{1}+s^{2} v\right)\right)$.

Hence, for any $c \in \mathcal{B}$ with $c \gg \theta$, there exists $N \in \mathbb{N}$ such that

$$
\rho\left(z_{*}, \mathcal{F}_{Z_{*}}\right) \preceq \tau_{1} \rho\left(z_{i+1}, z_{*}\right)+\tau_{2} \rho\left(z_{i}, z_{*}\right) \ll c .
$$

This implies, based on Lemma 2.12, that $\rho\left(z_{*}, \mathcal{F}_{Z_{*}}\right)=\theta$. Therefore, $\mathcal{F}_{z_{*}}=z_{*}$. This completes the proof. $\square$ Next example illustrates the above result. 
Example 3.4. Consider the algebra $\mathcal{B}=C_{\mathbb{R}}^{1}[0,1]$ with the norm

$$
\|z\|=\|z\|_{\infty}+\left\|z^{\prime}\right\|_{\infty} .
$$

Define on $\mathcal{B}$ a multiplication in the usual way. Then, $\mathcal{B}$ is a Banach algebra with unite $e=1$. If

$$
\mathcal{K}=\{z \in \mathcal{B}: z(x) \geq 0, x \in[0,1]\},
$$

then $\mathcal{K}$ is a solid cone which is not normal.

Suppose that $\mathcal{Z}=[0,1)$ and define a mapping $\rho: \mathcal{Z} \times \mathcal{Z} \rightarrow \mathcal{B}$ by

$$
\rho(z, y)(x)=|z-y|^{2} e^{x}, \text { for all } z, y \in \mathcal{Z} \text {. }
$$

Then $(\mathcal{Z}, \rho)$ is a complete cone $b$-metric space over Banach algebra $\mathcal{B}$. Let $\mathcal{F}: \mathcal{Z} \rightarrow \mathcal{Z}$ and $\alpha: \mathcal{Z} \times \mathcal{Z} \rightarrow \mathcal{B}$ be defined as follows:

$$
\mathcal{F}_{z}=\left\{\begin{array}{c}
\frac{\sqrt{5}}{3} z, \text { if } z \in[0,1] ; \\
z+1, \text { if } z>1,
\end{array} \quad \text { and } \alpha(z, y)=\left\{\begin{array}{l}
s, \text { if } z, y \in[0,1] ; \\
\theta, \text { elsewhere. }
\end{array} .\right.\right.
$$

Then, the mapping $\mathcal{F}$ is $\alpha$-admissible. In fact, let $z, y \in \mathcal{Z}$ such that $\alpha(z, y) \succeq s$. By definition of the mapping $\alpha$, it implies that $z, y \in[0,1]$. Therefore,

$$
\alpha\left(\mathcal{F}_{z}, \mathcal{F} y\right)=\alpha\left(\frac{\sqrt{5}}{3} z, \frac{\sqrt{5}}{3} y\right)=s .
$$

Also, there exists $z_{0}$ in $\mathcal{Z}$ such that $\alpha\left(z_{0}, \mathcal{F} z_{0}\right) \succeq s$. Indeed, for $z_{0}=1$, we have

$$
\alpha\left(z_{0}, \mathcal{F} z_{0}\right)=\alpha(1, \mathcal{F} 1)=\alpha\left(1, \frac{\sqrt{5}}{3}\right)=e
$$

Since $[0,1]$ is complete, then $(\mathcal{Z}, \rho)$ is $\alpha$-regular. Indeed, let $\left\{z_{i}\right\}$ be a sequence in $\mathcal{Z}$ such that $\alpha\left(z_{i}, z_{i+1}\right) \succeq s$ for all $i \in \mathbb{N}$ and let $z_{i} \rightarrow z \in \mathcal{Z}(i \rightarrow \infty)$. Then $\left\{z_{i}\right\} \subset[0,1]$ and $z_{i}+z \rightarrow 2 z$ $(i \rightarrow \infty)$. Therefore, $z_{i} \rightarrow z \quad(i \rightarrow \infty)$ in $(\mathcal{Z}, \rho)$. This implies that $z \in[0,1]$ and that $\alpha\left(z_{i}, z\right)=s$, for all $i \in \mathbb{N}$. Furthemore, by choosing $v_{1}=\frac{1}{9}+\frac{1}{9} x, v_{2}=\frac{1}{18}+\frac{1}{18} x$ and $v_{3}=\frac{1}{24}+\frac{1}{24} x$ in $\mathcal{K}$, simple calculations show that all the conditions of Theorem 3.3 are satisfied so there exists a fixed point $z=\theta$ of the mapping $\mathcal{F}$. $\square$

Now we state a theorem in which we shall establish the uniqueness of fixed point of a generalized Reich type contraction as follows:
Theorem 3.5. Let $(\mathcal{Z}, \rho)$ be a complete cone b-metric spaces over Banach algebra $\mathcal{B}$ with coefficient $s \geq 1, \mathcal{K}$ be the underlying solid cone and $\alpha: \mathcal{Z} \times \mathcal{Z} \rightarrow \mathcal{B}$ be a function. Suppose the mapping $\mathcal{F}: \mathcal{Z} \rightarrow \mathcal{Z}$ is a generalized Reich type contraction with vectors $v_{k} \in \mathcal{K}(k=1,2,3)$ such that $v_{1}$ commutes with $v_{2}+v_{3}$. Suppose that:

1) $\mathcal{F}$ is $\alpha$-admissible mapping;

2) there exists $z_{0}$ in $\mathcal{Z}$ such that $\alpha\left(z_{0}, \mathcal{F} z_{0}\right) \succeq s$;

3) either $\mathcal{F}$ is continuous or $(\mathcal{Z}, \rho)$ is $\alpha$-regular;

4) for any $z, y \in F i x(\mathcal{F})$, there exists $x \in \mathcal{Z}$ such that $\alpha(z, x) \succeq s$ and $\alpha(y, x) \succeq s$, where $F i x(\mathcal{F})$ denotes the set of all fixed points of $\mathcal{F}$,

then $\mathcal{F}$ has a unique fixed point $z_{*}$ in $\mathcal{Z}$.

PROOF. Following the same arguments to those in the proof of Theorem 3.3, we obtain the existence of fixed point.

To show that fixed point is unique, we take $z_{*}, y_{*} \in \operatorname{Fix}(\mathcal{F})$ such that $z_{*} \neq y_{*}$. Hence, by hypothesis 4 ), there exists $x \in \mathcal{Z}$ such that

$$
\alpha\left(z_{*}, x\right) \succeq s \text { and } \alpha\left(y_{*}, x\right) \succeq s .
$$

Since $\mathcal{F}$ is $\alpha$-admissible mapping and $z_{*}, y_{*} \in \operatorname{Fix}(\mathcal{F})$, from (15) we obtain

$$
\alpha\left(z_{*}, \mathcal{F}^{i} x\right) \succeq s \text { and } \alpha\left(y_{*}, \mathcal{F}^{i} x\right) \succeq s, \text { for all } i \in \mathbb{N} .
$$

Now, from (1) and (16), we get

$$
\begin{gathered}
\rho\left(z_{*}, \mathcal{F}^{i} x\right)=\rho\left(\mathcal{F}_{z_{*}}, \mathcal{F}\left(\mathcal{F}^{i} x\right)\right) \\
\preceq v_{1} \rho\left(z_{*}, \mathcal{F}^{i-1} x\right)+v_{2} \rho\left(z_{*}, \mathcal{F}_{z_{*}}\right)+v_{3} \rho\left(\mathcal{F}^{i-1} x, \mathcal{F}\left(\mathcal{F}^{i-1} x\right)\right) \\
\preceq v_{1} \rho\left(z_{*}, \mathcal{F}^{i-1} x\right)+s v_{3}\left[\rho\left(\mathcal{F}^{i-1} x, z_{*}\right)+\rho\left(z_{*}, \mathcal{F}^{i} x\right)\right], \text { i.e. } \\
\left(e-s v_{3}\right) \rho\left(z_{*}, \mathcal{F}^{i} x\right) \preceq\left(v_{1}+s v_{3}\right) \rho\left(z_{*}, \mathcal{F}^{i-1} x\right) .
\end{gathered}
$$

Similarly,

$$
\begin{gathered}
\rho\left(\mathcal{F}^{i} x, z_{*}\right)=\rho\left(\mathcal{F}\left(\mathcal{F}^{i} x\right), \mathcal{F}_{z_{*}}\right) \\
\preceq v_{1} \rho\left(\mathcal{F}^{i-1} x, z_{*}\right)+v_{3} \rho\left(z_{*}, \mathcal{F}_{z_{*}}\right)+v_{2} \rho\left(\mathcal{F}^{i-1} x, \mathcal{F}\left(\mathcal{F}^{i-1} x\right)\right) \\
\preceq v_{1} \rho\left(\mathcal{F}^{i-1} x, z_{*}\right)+s v_{2}\left[\rho\left(\mathcal{F}^{i-1} x, z_{*}\right)+\rho\left(z_{*}, \mathcal{F}^{i} x\right)\right], \text { i.e. } \\
\left(e-s v_{2}\right) \rho\left(\mathcal{F}^{i} x, z_{*}\right) \preceq\left(v_{1}+s v_{2}\right) \rho\left(\mathcal{F}^{i-1} x, z_{*}\right) .
\end{gathered}
$$

Adding up (17) and (18), we have

$$
\begin{aligned}
\left(2 e-s v_{2}-s v_{3}\right) \rho\left(z_{*}, \mathcal{F}^{i} z_{*}\right) \preceq\left(2 v_{1}+s v_{2}+s v_{3}\right) \rho\left(z_{*}, \mathcal{F}^{i-1} x\right) \\
(2 e-s v) \rho\left(z_{*}, \mathcal{F}^{i} x\right) \preceq\left(2 v_{1}+s v\right) \rho\left(z_{*}, \mathcal{F}^{i-1} x\right) \\
\rho\left(z_{*}, \mathcal{F}^{i} x\right) \preceq(2 e-s v)^{-1}\left(2 v_{1}+s v\right) \rho\left(z_{*}, \mathcal{F}^{i-1} x\right), \text { i.e. }
\end{aligned}
$$




$$
\rho\left(z_{*}, \mathcal{F}^{i} x\right) \preceq \tau \rho\left(z_{*}, \mathcal{F}^{i-1} x\right),
$$

where $\tau=(2 e-s v)^{-1}\left(2 v_{1}+s v\right)$. Hence,

$$
\begin{aligned}
\rho\left(z_{*}, \mathcal{F}^{i} x\right) & \preceq \tau \rho\left(z_{*}, \mathcal{F}^{i-1} x\right) \\
& \preceq \tau^{2} \rho\left(z_{*}, \mathcal{F}^{i-2} x\right) \\
& \vdots \\
\rho\left(z_{*}, \mathcal{F}^{i} x\right) & \preceq \tau^{i} \rho\left(z_{*}, x\right), \text { for all } i \in \mathbb{N} .
\end{aligned}
$$

Since $\delta(\tau)<1$, by Remark 2.10, it follows that $\left\|\tau^{i}\right\| \rightarrow 0(i \rightarrow \infty)$ and so

$$
\left\|\tau^{i} \rho\left(z_{*}, x\right)\right\| \leq\left\|\tau^{i}\right\|\left\|\rho\left(z_{*}, x\right)\right\| \rightarrow 0(i \rightarrow \infty) .
$$

Therefore, based on Lemma 2.6, we conclude that for any $c \in \mathcal{B}$ with $c \gg \theta$, there exists $N \in \mathbb{N}$ such that

$$
\rho\left(z_{*}, \mathcal{F}^{i} x\right) \preceq \tau^{i} \rho\left(z_{*}, x\right) \ll c \text { for all } i \in \mathbb{N} .
$$

Hence, $\mathcal{F}^{i} x \rightarrow z_{*}(i \rightarrow \infty)$. Similarly, we get that $\mathcal{F}^{i} x \rightarrow y_{*}$ $(i \rightarrow \infty)$.

Then, by uniquieness of the limit, we have $z_{*}=y_{*}$. The theorem is thus proved. $\square$

The next theorem is an ordered version of generalized Riech type contraction on cone $b$-metric space with Banach algebra.

Theorem 3.6. Let $(\mathcal{Z}, \sqsubseteq)$ be a partially ordered set, let $(\mathcal{Z}, \rho)$ be a complete cone b-metric spaces over Banach algebra $\mathcal{B}$ with coefficient $s \geq 1$ and $\mathcal{K}$ be the underlying solid cone. Let $\mathcal{F}: \mathcal{Z} \rightarrow \mathcal{Z}$ be continuous non-decreasing mapping with respect to $\sqsubseteq$. Assume that the following conditions are valid:

1) there exists vectors $v_{k} \in \mathcal{K} \quad(k=1,2,3)$ such

$$
\begin{aligned}
& 2 s \delta\left(v_{1}\right)+(s+1) \delta\left(v_{2}+v_{3}\right)<2 \text { and } \\
& \rho\left(\mathcal{F}_{z}, \mathcal{F} y\right) \preceq v_{1} \rho(z, y)+v_{2} \rho\left(z, \mathcal{F}_{z}\right)+v_{2} \rho(y, \mathcal{F} y),
\end{aligned}
$$

for all $z, y \in \mathcal{Z}$ with $z \sqsubseteq y$;

2) there exists $z_{0} \in \mathcal{Z}$ such that $z_{0} \sqsubseteq \mathcal{F} z_{0}$,

then $\mathcal{F}$ has a unique fixed point $z_{*}$ in $\mathcal{Z}$.

ProOF. Let $\alpha_{j}: \mathcal{Z} \times \mathcal{Z} \rightarrow \mathcal{B}$ be function defined by

$$
\alpha_{j}(z, y)=\left\{\begin{array}{cc}
s, & \text { if } z \sqsubseteq y ; \\
\theta, & \text { elsewhere. }
\end{array}\right.
$$

Note that condition 1) implies that the mapping $\mathcal{F}$ is a generalized Reich contraction. Since $\mathcal{F}$ is non-decreasing mapping it follows that it is also $\alpha_{j}$-admissible mapping. The condition 2) implies that there exists $z_{0} \in \mathcal{Z}$ such that $\alpha_{j}\left(z_{0}, \mathcal{F} z_{0}\right)=s$. Therefore, since $\mathcal{F}$ is continuous, all the conditions of Theorem 3.3 are satisfied, we conclude that the mapping $\mathcal{F}$ has a fixed point in $\mathcal{Z}$. This completes the proof.

Now we deduce many existing results in the mention literature for metric, cone metric and cone $b$-matric spaces, as follows:

Let $\alpha: \mathcal{Z} \times \mathcal{Z} \rightarrow \mathcal{B}$ be the function defined by $\alpha(x, y) \geq 1$, for all $z, y \in \mathcal{Z}$.Then

- Theorems 3.1, 3.2 and 3.4 in Hussain at al. (2017) are special cases of our Theorems 3.3 and 3.5, respectively, with $v_{2}=v_{3}=\theta$.

- Theorems 3.1, 3.2 and 3.5 in Malhotra et al. (2015) are special cases of our Theorems 3.3 and 3.5, respectively, with $v_{1}=1, v_{2}=v_{3}=\theta$.

- Theorems 3.1, 3.2 and 3.3 in Malhotra et al. (2017) are special cases of our Theorems 3.3 and 3.5, respectively, with $s=1, v_{1}=\theta, v_{2}=v_{3}$.

Let $\alpha: \mathcal{Z} \times \mathcal{Z} \rightarrow \mathcal{B}$ be function defined by $\alpha(x, y)=1$ for all $z, y \in \mathcal{Z}$. Then,

- Theorems 3.1 and 3.2 in Xu and Radenović (2015) are special case of our Theorem 3.5, with $s=1, v_{2}=v_{3}=\theta$ and $v_{1}=\theta, v_{2}=v_{3}$, respectively.

- $\quad$ Theorems 2.1 and 2.2 in Liu and $\mathrm{Xu}$ (2013) are special cases of our Theorem 3.5 with normal cone $\mathcal{K}$, with $s=1, v_{2}=v_{3}=\theta$ and $v_{1}=\theta, v_{2}=v_{3}$, respectively.

- Theorems 1 and 2 in Riech (1971) are special case of our Theorem 3.5 with a metric space $(\mathcal{Z}, \rho)$ where $\delta\left(v_{1}\right)=v_{1}$ for all $v_{1} \in \mathbb{R}^{+}$.

Let the function $\alpha_{j}: \mathcal{Z} \times \mathcal{Z} \rightarrow \mathcal{B}$ be define by $\alpha_{j}(z, y)=1$ for all $z, y \in \mathcal{Z}$.

- Theorems 2.1 and 2.2 in Nieto and Rodríguez-López (2005) are special cases of our Theorems 3.6 with a metric space $(\mathcal{Z}, \rho)$, where $\delta\left(v_{1}\right)=v_{1}$ for $v_{1} \in \mathbb{R}^{+}, s=1$ and $v_{2}=v_{3}=\theta$.

\section{CONCLUSION}

In this paper we introduced the notion of generalized Riech type $\alpha$-admissible mappings on cone metric space over Banach algebra and we prove three fixed point theorems for those contractions. We notice that our results are actual generalization of the recent results of Liu and Xu (2013), Xu and Radenović (2015), Malhotra et al. (2015), Hussain et al. (2017), Riech (1971), Nieto and Rodríguez-López (2005) and many known results in the literature. 


\section{ACKNOWLEGMENTS}

The first author is supported by Serbian Ministry of Science and Technology (TR 35030).

\section{REFERENCES}

Du, W. 2010. A note on cone metric fixed point theory and its equivalence. Nonlinear Analysis: Theory, Methods and Applications, 72(5), pp. 2259-2261. doi:10.1016/j.na.2009.10.026

Huang, L., \& Zhang, X. 2007. Cone metric spaces and fixed point theorems of contractive mappings. Journal of Mathematical Analysis and Applications, 332(2), pp. 14681476. doi:10.1016/j.jmaa.2005.03.087

Huang, H., \& Radenović, S. 2016. Some fixed point results of generalized Lipschitz mappings on cone b-metric spaces over Banach algebras. J. Computational Analysis and Applications, 20(3), pp. 566-583.

Huang, H., \& Radenović, S. 2015. Common fixed point theorems of generalized Lipschitz mappings in cone b-metric spaces over Banach algebras and applications. Journal of Nonlinear Sciences and Applications, 8(5), pp. 787-799. doi:10.22436/jnsa.008.05.29

Huang, H., \& Radenović, S. 2015. Common fixed point theorems of generalized Lipschitz mappings in cone metric spaces over Banach algebras. Appl. Math. Inf. Sci., 9(6). pmid:2990

Huang, H., \& Xu, S. 2013. Fixed point theorems of contractive mappings in cone b-metric spaces and applications. Fixed Point Theory and Applications, 2013(1), p. 112. doi:10.1186/1687-1812-2013-112

Huang, H., Radenovic, S., \& Deng, G. 2017. A sharp generalization on cone b-metric space over Banach algebra. The Journal of Nonlinear Sciences and Applications, 10(02), pp. 429-435. doi:10.22436/jnsa.010.02.09

Hussain, N., Al-solami, A. M., \& Kutbi, M. A. 2017. Fixed points of $\alpha$-admissible mappings in cone b-metric spaces with Banach algebra. Journal of Mathematical Analysis, 8(2), pp. 89-97.
Kadelburg, Z., Radenović, S., \& Rakočević, V. 2011. A note on the equivalence of some metric and cone metric fixed point results. Applied Mathematics Letters, 24(3), pp. 370-374. doi:10.1016/j.aml.2010.10.030

Kadelburg, Z., \& Radenović, S. 2013. A note on various types of cones and fixed point results in cone metric spaces. Asian Journal of Mathematics and Applications, Article ID amma0104, 7 pages.

Liu, H., \& Xu, S. 2013. Cone metric spaces with Banach algebras and fixed point theorems of generalized Lipschitz mappings. Fixed Point Theory and Applications, 2013(1), p. 320. doi:10.1186/1687-1812-2013-320

Malhotra, S. K., Sharma, J. B., \& Shukla, S. 2015. Fixed points of $\alpha$-admissible mappings in cone metric spaces with Banach algebra. International Journal of Analysis and Applications, 9(1), pp. 9-18.

Malhotra, S. K., Sharma, J. B., \& Shukla, S. 2017. Fixed points of generalized Kannan type $\alpha$-admissible mappings in cone metric spaces with Banach algebra. Theory and Applications of Mathematics and Computer Science, 7(1), pp. 1-13.

Nieto, J. J., \& Rodríguez-López, R. 2005. Contractive Mapping Theorems in Partially Ordered Sets and Applications to Ordinary Differential Equations. Order, 22(3), pp. 223-239. doi:10.1007/s11083-005-9018-5

Reich, S. 1971. Some remarks concerning contraction mappings. Canadian Mathematical Bulletin, 14, pp. 121-124. doi:10.4153/cmb-1971-024-9

Rudin, W. 1991. Functional Analysis.New York: McGraw-Hill. $2^{\text {nd }}$ edition.

Samet, B., Vetro, C., \& Vetro, P. 2012. Fixed point theorems for -contractive type mappings. Nonlinear Analysis: Theory, Methods and Applications, 75(4), pp. 2154-2165. doi:10.1016/j.na.2011.10.014

$\mathrm{Xu}$, S., \& Radenović, S. 2015. Fixed point theorems of generalized Lipschitz mappings on cone metric spaces over Banach algebras without assumption of normality. Fixed Point Theory and Applications, 2014(1), p. 102. doi:10.1186/1687-1812-2014-102 